\title{
Immunoblotting can help the diagnosis of ocular toxoplasmosis
}

D O Ho-Yen, D J Chapman, D Ashburn

\begin{abstract}
Aims-To determine whether IgG immunoblotting can improve the diagnosis of ocular toxoplasmosis.

Methods-Samples of serum were tested from patients with ocular lesions that could be caused by toxoplasmosis. All such samples from Scotland and Northern Ireland are usually referred to the Scottish Toxoplasma Reference Laboratory. From questionnaires filled out by the clinicians, two groups of sera were identified: ocular toxoplasmosis (active and quiescent), $\mathrm{n}=54$ (group 1); and eye disease as a result of other causes, $n=36$ (group 2). Control groups were made up of sera from patients with no eye disease and a normal dye test result ( $\leqslant 125 \mathrm{IU} / \mathrm{ml}), \quad n=16$ (group 3); and toxoplasma seronegative, cytomegalovirus (CMV) positive, and herpes simplex virus (HSV) positive sera (group 4), $\mathbf{n}=18$.
\end{abstract}

Results-Immunoblots with an active pattern could be identified (IgG antibodies against at least four antigens with molecular weight of $6,20,22,23,25$, and $36 \mathrm{kDa}$ ). Significantly more of this pattern was found in group 1 (33 of $54 ; 61.1 \%)$ compared with group 2 (nine of $36 ; 25 \%$ ) or group 3 (six of $16 ; 37.5 \%$ ). Within group 1 , significantly more sera with an active pattern had dye test results $\geqslant 65 \mathrm{IU} / \mathrm{ml}$ compared with those without. More sera from patients $<30$ years of age were found with the active pattern in group $1 \mathrm{com}-$ pared with group 2 . No group 4 sera had active immunoblot patterns.

Conclusions-The immunoblot result adds more support to the diagnosis of ocular toxoplasmosis. In cases where the clinical diagnosis is difficult, immunoblots are particularly indicated; if negative, other causes of eye disease should be sought.

(f Clin Pathol: Mol Pathol 2000;53:155-158)

Scottish Toxoplasma

Reference Laboratory,

Microbiology

Department,

Raigmore Hospital,

Inverness IV2 3UJ, UK

D O Ho-Yen

D J Chapman

D Ashburn

Correspondence to: Dr Ho-Yen

Accepted for publication 15 February 2000

email: microbiology@

raigmore.scot.nhs.uk
Keywords: ocular toxoplasmosis; IgG immunoblotting; dye test retinochoroiditis in the world. ${ }^{1}$ Conventionally, it is believed that most cases are the result of congenital toxoplasma infection, ${ }^{2}$ with only a few being acquired infections. ${ }^{3}$ Although there may be a suggestion that ocular toxoplasmosis is only a clinical diagnosis, ${ }^{4}$ laboratory investigation is frequently undertaken. This is especially important because the clinical picture can be complicated and there are many other causes of retinochoroiditis. $^{34}$
Toxoplasma gondii is the most common cause of
The difficulty with the serological diagnosis of ocular toxoplasmosis is that toxoplasma specific antibody is not usually high. ${ }^{2}$ This is believed to be because most cases of ocular toxoplasmosis are congenital and the neonate's immature immune system does not produce high antibody concentrations. ${ }^{5}$ Therefore, other investigators have looked for toxoplasma specific antibodies in aqueous humour. ${ }^{4}$ However, this latter approach is not generally used, and most clinicians rely on a clinical diagnosis with the presence of toxoplasma specific antibody to support the diagnosis. ${ }^{245}$ Predictably, this approach has been criticised as producing an overdiagnosis of ocular toxoplasmosis. $^{56}$

We have shown previously that there is a direct relation between dye test results and ocular disease. ${ }^{6}$ We believe that a dye test result of $\geqslant 65 \mathrm{IU} / \mathrm{ml}$ in the presence of appropriate clinical findings is indicative of ocular toxoplasmosis, and with lower results alternative causes of the ocular lesions should be sought. ${ }^{6}$ Our present study extends the previous work, and all samples with sufficient sera were tested by immunoblotting. Because we have shown previously that IgG immunoblotting can identify active toxoplasma infection, ${ }^{7}$ our present study was designed to see whether the presence of an active IgG immunoblot pattern is more helpful in diagnosis than specific toxoplasma antibody.

\section{Methods}

The Scottish Toxoplasma Reference Laboratory receives specimens from laboratories throughout Scotland and Northern Ireland. For those patients with ocular disease and a positive dye test result ( $\geqslant 2 \mathrm{IU} / \mathrm{ml}$ ), clinicians are sent questionnaires to obtain information about eye disease. Patients were grouped as follows: group 1 comprised 54 patients clinically diagnosed with ocular toxoplasmosis (36 of 54, with active lesions and 18 of 54, with quiescent lesions); group 2 comprised 36 patients with eye disease as a result of other causes; group 3 was a control group of 16 patients without eye disease who had a normal dye test result as determined using seropositive patients without clinical evidence of active infection; and group 4 was a further control group of toxoplasma specific antibody negative sera from six patients without ocular disease, six cytomegalovirus (CMV) positive sera (CMV titre $\geqslant 64$ ), and six herpes simplex virus (HSV) positive sera (HSV titre $\geqslant 64$ ). Toxoplasma specific antibodies were measured in all sera using sensitive in house screening tests for $\operatorname{IgG}^{8}$ and $\operatorname{IgM},{ }^{9}$ and were confirmed using a micromodification of the Sabin- 
Table 1 Individual antigens recognised by antibodies in sera from patients in the four different groups and the number with an active pattern (defined as recognition of four or more antigens)

\begin{tabular}{|c|c|c|c|c|c|c|c|c|c|c|}
\hline & \multirow[b]{2}{*}{$n$} & \multirow{2}{*}{$\begin{array}{l}\text { No (\%) with } \\
D T \geqslant 65 \mathrm{IU} / \mathrm{ml}\end{array}$} & \multirow{2}{*}{$\begin{array}{l}\text { No (\%) with } \\
D T \geqslant 125 \\
I U / m l\end{array}$} & \multicolumn{6}{|c|}{ No sera $(\%)$ with antibodies against antigens with molecular weight $(k D a)$} & \multirow{2}{*}{$\begin{array}{l}\text { No (\%) } \\
\text { with active } \\
\text { immunoblot } \\
\text { pattern }\end{array}$} \\
\hline & & & & 6 & 20 & 22 & 23 & 25 & 36 & \\
\hline Group 1: ocular toxoplasmosis & 54 & $35(65)$ & $6(11)$ & $33(61)$ & $42(78)$ & $47(87)$ & $23(43)$ & $35(65)$ & $32(59)$ & $33(61)$ \\
\hline Group 2: other eye disease & 36 & $10(28)$ & $2(6)$ & $9(25)$ & $16(44)$ & $20(56)$ & $15(42)$ & $10(28)$ & $10(28)$ & $9(25)$ \\
\hline Group 3: past infection & 16 & $8(50)$ & $4(25)$ & $9(56)$ & $12(75)$ & $5(31)$ & $5(31)$ & $4(25)$ & $5(31)$ & $6(38)$ \\
\hline Group 4: control & 18 & $0(0)$ & $0(0)$ & $0(0)$ & $1(6)$ & $0(0)$ & $0(0)$ & $3(17)$ & $0(0)$ & $0(0)$ \\
\hline
\end{tabular}

DT, dye test.

Feldman dye test. ${ }^{10}$ Screen IgM positive results were confirmed using a commercial IgM capture enzyme linked immunosorbent assay (Toxonostika ELISA IgM II; Organon Teknika, Cambridge, UK). Those sera with negative ELISA IgM were also tested using a more sensitive $\operatorname{IgM}$ immunosorbent agglutination assay (Toxo-ISAGA; BioMerieux, Basingstoke, $\mathrm{UK})$.

IgG immunoblotting was performed as described previously. ${ }^{7}$ Briefly, antigen was prepared using peritoneal exudates from cotton rats infected with the $\mathrm{RH}$ strain of toxoplasma. Tachyzoites were washed with sterile normal saline, frozen and thawed, and then sonicated. Before use, antigen was mixed with an equal volume of lysis buffer containing $4 \%$ lauryl sulphate and 2\% 2 mercaptoethanol. Proteins were separated by sodium dodecyl sulphate polyacrylamide gel electrophoresis (SDSPAGE), ${ }^{11}$ using a $15 \%$ separation gel and a 3\% stacking gel. Antigen was boiled for five minutes and added to a single well, $125 \mathrm{~mm}$ long.

Mixtures of molecular weight markers, either prestained (Sigma Chemical Co Ltd, Poole, Dorset, UK) or unstained (Sigma Dalton Mark VII-L) were added to two separate wells. Gels were electrophoresed overnight at $8 \mathrm{~mA} / \mathrm{gel}$ and proteins were transferred to a nitrocellulose membrane at $300 \mathrm{~mA} / \mathrm{gel}$ for four hours. The nitrocellulose membrane was blocked for one hour with $5 \%$ non-fat milk in phosphate buffered saline (PBS; $\mathrm{pH}$ 7.2) then rinsed in PBS. After drying, the membrane was stored in the dark at room temperature until use. The membranes were cut into strips approximately $3 \mathrm{~mm}$ wide and incubated overnight with serum diluted $1 / 50$ in 5\% non-fat milk in PBS/ 0.05\% Tween-20 (PBST). Strips were washed in five changes of PBST and conjugate was added (goat antihuman IgG; Sigma). After two hours, strips were washed with four changes of PBST and once with PBS alone. Substrate (4-chloronaphthol in methanol, hydrogen peroxide in PBS) was added for 10 minutes, after which strips were washed twice with PBS, twice with distilled water, and then dried. Statistical analysis was performed using the $\chi^{2}$ test.

\section{Results}

Toxoplasma specific antibodies were detected in all sera from patient groups 1 and 2 and control group 3. Dye test results ranged from 8 to $500 \mathrm{IU} / \mathrm{ml}$. Specific toxoplasma ISAGA IgM was detected in only one patient from group 1, who also had a dye test result of $250 \mathrm{IU} / \mathrm{ml}$, which suggests acquired infection. All other sera were IgM negative. Our normal range for the dye test is $\leqslant 125 \mathrm{IU} / \mathrm{ml}$ and only six of 54 group 1 sera and two of 36 group 2 sera had raised results. No group 3 sera had dye test results $>125 \mathrm{IU} / \mathrm{ml}$ (table 1 ). Table 1 shows the antigens recognised by antibodies in sera from patients in different groups as measured with immunoblotting. Detection of four or more of the $6,20,22,23,25$, or $36 \mathrm{kDa}$ antigens was consistent with active infection (fig 1). In group 1, 33 of 54 patients had an active immunoblot pattern, compared with only nine of 36 patients from group 2 $(\mathrm{p}<0.0001)$. There was also a difference in distribution of dye test results between the two groups (table 1); more patients in group 1 had results $\geqslant 65 \mathrm{IU} / \mathrm{ml}$ compared with group 2 $(p<0.0001)$. The number of antigens recognised in immunoblotting and their intensity of staining is related to the dye test result, and within group 1 a higher proportion of patients with an active immunoblot pattern had dye test results $\geqslant 65 \mathrm{IU} / \mathrm{ml}$ than $\leqslant 30 \mathrm{IU} / \mathrm{ml}$ $(\mathrm{p}<0.01)$. An active pattern was recorded in the one group 1 ISAGA IgM positive patient (who had a dye test result of $250 \mathrm{IU} / \mathrm{ml}$ ).

No group 3 patients were specific IgM positive, and all had normal dye test results $(\leqslant 125 \mathrm{IU} / \mathrm{ml}$ ), but six of $16 \mathrm{had}$ an active immunoblot pattern. However, the proportion of group 1 patients with this pattern was higher than in group $3(\mathrm{p}<0.005)$. There was no difference between the proportion of patients in groups 2 and 3 with the active immunoblot pattern ( $p>0.05)$, suggesting that these patients were from the same populations. Group 4 sera were all toxoplasma specific antibody negative. A single band representing antigens with molecular weights 20 or $25 \mathrm{kDa}$ was recorded in four of six CMV positive sera only. Therefore, none of the sera from group 4 had an active immunoblot pattern. Further analysis of active immunoblot pattern by patient age revealed that a higher population of group 1 patients $\leqslant 30$ years old had an active pattern compared with group $2(\mathrm{p}<0.05)$, which is consistent with ocular toxoplasmosis manifesting at a younger age compared with primary infection, which was found more frequently in people over 30 years of age.

\section{Discussion}

It is always a challenge for a laboratory to improve tests in conditions that are essentially clinical diagnoses. Such is the case with ocular toxoplasmosis. Established dogma is that active ocular toxoplasmosis and the value of the dye test do not correlate. ${ }^{12}$ While our previous 


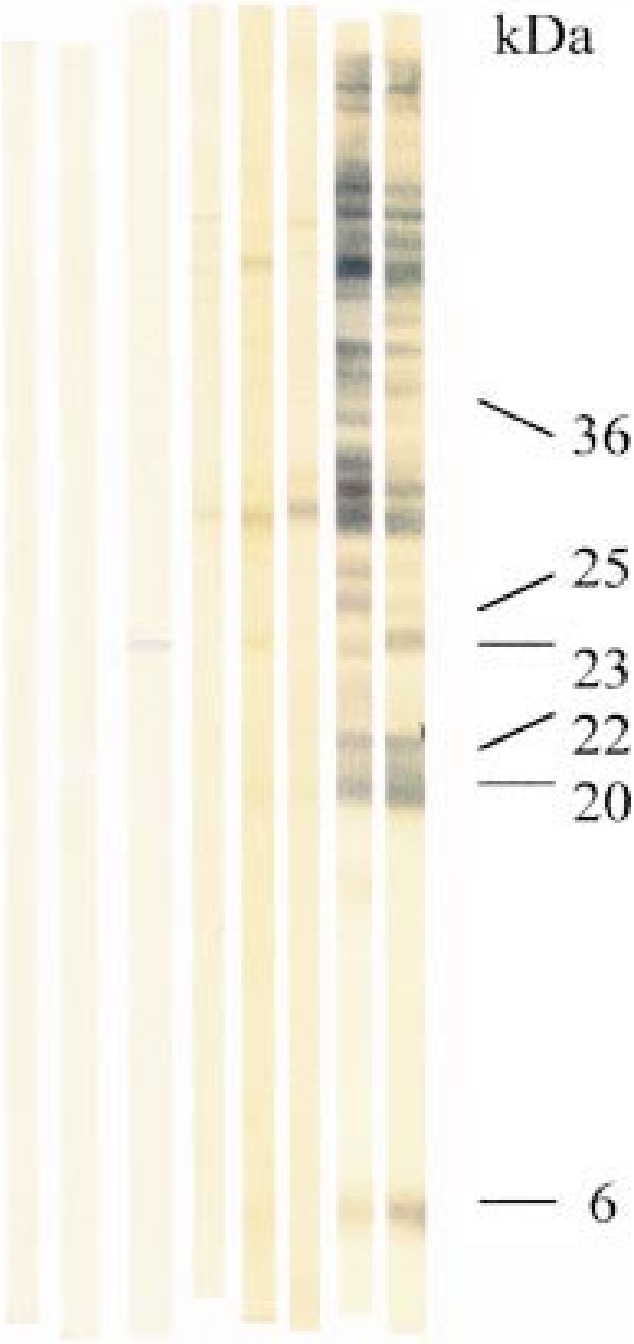

123345678

Figure 1 Toxoplasma antigens recognised by $\operatorname{IgG}$ antibodies in sera. Lane 1, negative control; lanes 2 and 3, cytomegalovirus $(C M V)$ positive sera (lane 2 shows a single band at $20 \mathrm{kDa}$, lane 3 has a single band at $25 \mathrm{kDa}$ ); lanes 4-6, non-active pattern (lane 4 has no relevant bands, lane 5 has weakly stained bands at 6, 20, and $23 \mathrm{kDa}$, and lane 6 has a weakly stained band at $20 \mathrm{kDa}$ ); lanes 7 and 8, active pattern, ( $\geqslant 4$ bands at 6 , $20,22,23,25$, and $36 \mathrm{kDa}$ ).

results ${ }^{6}$ showed this to be incorrect, our current results show significant differences in the immunoblot patterns seen with sera from patients with ocular toxoplasmosis compared with sera from patients with other causes of their eye disease $(\mathrm{p}<0.001)$.

Immunoblotting is an important technique for improving toxoplasma diagnosis. The presence of antibodies against low molecular weight antigens reflects active infection and can be particularly useful in reactivated toxoplasmosis. ${ }^{7}$ Even if the dye test is within the normal range, this active pattern can be diagnostic. Our finding of such a pattern in 33 of 54 sera from patients with suspected ocular toxoplasmosis supports this diagnosis. Group 1 comprised patients with active and quiescent lesions. The active immunoblot pattern was present in sera from both subgroups but the proportions did not differ significantly. This is not unexpected because active lesions can become quiescent as early as four weeks after onset. ${ }^{13}$ Many of our sera were not collected until several months after onset, so these were treated as one group. Similarly, this active pattern was present in nine of 36 patients in whom other causes of eye disease were suspected, but the results suggest that in these patients the clinical diagnosis might have been wrong, and that it might have been ocular toxoplasmosis. It is possible that in early acquired infection, the active immunoblot pattern might not have developed; therefore, patients should be retested two weeks after onset. In both groups, sera without this pattern should prompt the search for other causes of the eye disease.

Among the patients with ocular toxoplasmosis (group 1), a higher proportion of sera with an active immunoblot pattern had dye test results $\geqslant 65 \mathrm{IU} / \mathrm{ml}$, compared with sera with a non-active pattern $(\mathrm{p}<0.01)$. These results are consistent with the suggestion that a dye test result $\geqslant 65 \mathrm{IU} / \mathrm{ml}$ is found in patients with ocular toxoplasmosis. ${ }^{6}$ The proportion of patients greater than 30 years with an active immunoblot pattern was higher in sera from patients with suspected ocular toxoplasmosis compared with sera from patients with possible other causes of eye disease. This is consistent with most cases of ocular toxoplasmosis being congenital and presenting at younger ages. ${ }^{3}$ Nevertheless, positive IgM results in one group 1 patient may indicate primary infection, confirming suggestions that ocular toxoplasmosis can also result from acquired infection. ${ }^{3}$ The presence of an active immunoblot pattern with normal dye test and negative $\operatorname{IgM}$ results in our control group suggests that this pattern may persist for many weeks. This is consistent with our previous finding that this pattern predominates in the first 16 weeks of primary infection, and may persist for up to 36 weeks. $^{7}$ As always, it is therefore crucially important that laboratory results are related to the clinical findings. Nevertheless, with most ocular toxoplasmosis, reactivated infection occurs in the first 2-3 decades of life, ${ }^{3}$ and therefore persistence of the active immunoblot pattern should not be a problem.

Historically, the diagnosis of ocular toxoplasmosis has been greatly influenced by whether the condition reflects congenital or acquired infection. ${ }^{3}$ With congenital infection, the dye test result may be as low as $2 \mathrm{IU} / \mathrm{ml}$ and $\mathrm{IgM}$ is not frequently present. ${ }^{6}$ With acquired infection, diagnosis is easy because one expects high dye test results and the presence of $\operatorname{IgM}$. Recently, more and more cases of ocular toxoplasmosis following acquired infection have been identified. ${ }^{2}$ These findings and our results suggest that there should be a different approach to the diagnosis of ocular toxoplasmosis. A dye test of $\geqslant 65 \mathrm{IU} / \mathrm{ml}$ is indicative of the condition and immunoblots should be performed. Low dye test results or a non-active immunoblot pattern should prompt the clinician to look for other causes of the eye disease. 
We are grateful to the clinicians and laboratories who use the Scottish Toxoplasma Reference Laboratory. We are funded by the national services division of the Scottish Office. We would like to than
manuscript.

1 Pavesio CE, Lightman S. Toxoplasma gondii and ocular toxoplasmosis: pathogenesis. $\mathrm{Br} \mathcal{F}$ Ophthalmol 1996;80 1099-107.

2 Montoya JG, Remington JS. Toxoplasmic chorioretinitis in the setting of acute acquired toxoplasmosis. Clin Infect Dis 1996;23:277-82.

3 Ho-Yen DO. Clinical features. In: Ho-Yen DO, Joss AWL eds. Human toxoplasmosis. Oxford: Oxford University Press, 1992:56-78.

4 Bornand JE, de Gottran P. Uveitis: is ocular toxoplasmosis only a clinical diagnosis? Ophthalmologica 1997;211: only a $87-9$.

5 Swanson MW. Association of antibody titer and chorioretinal scarring in toxoplasmosis retinochoroiditis. $\mathcal{F}$ Am Optom Assoc 1989;60:735-40.
6 Chapman DJ, Ashburn D, Ogston SA, et al. The relationship between ocular toxoplasmosis and levels of specific toxoplas

mosis antibodies. Epidemiol Infect 1999;122:299-303.
7 Ashburn D, Davidson MM, Joss AWL, et al. Improved diagnosis of reactivated toxoplasmosis. F Clin Pathol: Mol Pathol 1998;51:105-9.

8 Joss AWL, Skinner LJ, Chatterton JMW, et al. Toxoplasmosis: effectiveness of enzyme immunoassay screening. Med Lab Sci 1989;46:107-12.

9 Joss AWL, Skinner LJ, Moir IL, et al. Biotin-labelled antigen screening test for toxoplasmosis IgM antibody. $\mathcal{F}$ Clin Pathol 1989;42:206-9

10 Williams KAB, Scott JM, Macfarlane DE, et al. Congenital toxoplasmosis: a prospective survey in the west of Scotland. f Infect 1981;3:219-29.

11 Laemmlli UK. Cleavage of structural proteins during the assembly of the head of bacteriophage T4. Nature 1970;227:680-5.

12 Rothova A, van Knappen F, Baarsma GS, et al. Serology in ocular toxoplasmosis. Br f Ophthalmol 1986;70:615-22.

13 Dutton GN. Toxoplasmic retinochoroiditis-a historical review and current concepts. Ann Acad Med Singapore 1989;18:214-21. 